\title{
Analysis of a tractable model for combustion instability: the effect of delay and low pass filtering
}

\author{
F. Bouziani, I.D. Landau, R.R. Bitmead ${ }^{\dagger}$, A. Voda-Besançon \\ Laboratoire d'Automatique de Grenoble, ENSIEG BP 46, 38402 Saint-Martin d'Hères, France \\ $\dagger$ Department of Mechanical \& Aerospace Engineering, University of California, San Diego, La Jolla CA 92093-0411, USA
}

\begin{abstract}
In this paper a new analytically tractable model for combustion instability is proposed. The model is based on two coupled resonators in a feedforward path, and a feedback path composed of a delay, generalized Van der Pol term and a low pass filter. The model is analyzed and approximated using a refined Krylov-Bogoliubov (K-B) method. The analysis shows that the model captures the phenomenon of two coexisting oscillating modes which has been noted in combustion instability in [1], [2]. Conditions for the occurrence of various operation regimes have been established. The importance of delay and low pass filtering is discussed in this article. A frequency domain comparison between K-B approximations and the true outputs of the model has been provided in the end of the paper.
\end{abstract}

Index Terms-modelling, combustion instability, nonlinear oscillating systems, Krylov-Bogoliubov method.

\section{INTRODUCTION}

Combustion instabilities constitute a major problem for combustion turbomachinery such as jet engines and gas turbine powerplants, when operating in lean premixed combustion, which is desirable from the standpoint of minimizing pollution. Research programs in this area are actively conducted in a number of countries [3], [4], [5], [6], [7], [8]. The modeling of this instability is difficult and somewhat contentious in detail, with the broad explanation being the positive feedback coupling between the thermal heat-release process and the acoustics of the combustion chamber. The phenomenon has been experimentally known for more than 200 years.

Several control strategies have been proposed to deal with this instability, see [9] for an overview. One of most effective strategies, successfully experimented, is active control with the actuation taking place primarily through modulation of a fraction of the fuel flow into the combustion chamber [10], [11]. The goal of active control is to modify the combustion system dynamics. The systematic implementation and reliable design of active control requires the development of a realistic low order model which exhibits the dominant dynamical effects as limit cycles. The phenomenon has been scientifically studied in the laboratory since Rayleigh and Rijke [12], [13] and has been described much earlier in [14]. However, a low order and accurate modelling of such a complex system is an extremely challenging task and is still not fully resolved.

In [15], Peracchio and Proscia have proposed a low order model resulting from several physical investigations, the model was characterized by a linear resonator in feedback

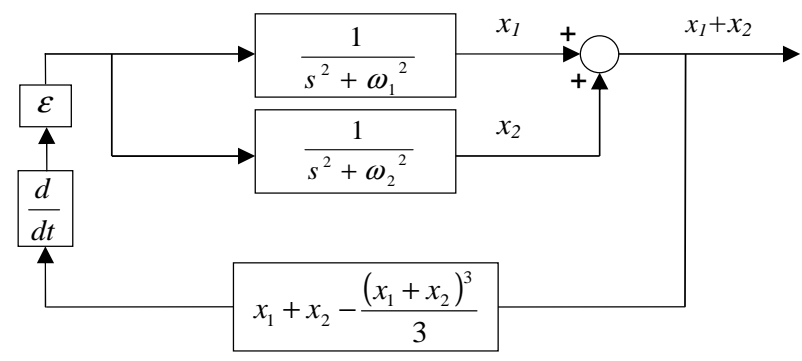

Fig. 1. Combustion instability model based on coupled Van der Pol equations

with a delay and a nonlinear static function. In order to explain the coexistence of two modes (see [2], [16]), the model has been extended in [1] by adding a second resonator. The model in [1] features in the feedback loop a cascade of a : differentiator - delay - static nonlinear characteristic - low pass filter - differentiator. An analytically tractable model for [1] has been proposed in [17] based on coupled Van der Pol equations. The block diagram of the model is shown in Figure 1, where $\omega_{1}$ and $\omega_{2}$ are the natural radian frequencies of the first and second resonators respectively and which can have arbitrary values with some modest provisions to be developed, $\varepsilon$ is a small positive quantity. The model has been successfully analyzed using Krylov-Bogoliubov approach in [17]. However, this model does not include the cascade differentiator - delay and the low pass filter. While interesting results have been obtained concerning the existence or quenching of the oscillations in the system, the model was not able to explain the simultaneous presence of two oscillating modes observed experimentally, when the frequencies of the two resonators are in ratio different from 1,3 and $\frac{1}{3}$. The present paper which is the continuation of [17] considers an analytically tractable model which takes into account

1) The generalization of the nonlinear static characteristic (see [1], previously approximated by basic Van der Pol characteristic).

2) The existence of time delay plus differentiator block and low pass filtering in the feedback path (see [15]).

The first point suggests replacing in the model the basic Van der Pol term $\varepsilon \frac{d}{d t}\left(y-\frac{1}{3} y^{3}\right)$ by a generalized Van der Pol term $\frac{d}{d t}\left(\varphi_{v 0}+\varphi_{v 1} y-\frac{\varphi_{v 3}}{3} y^{3}\right)$, where $\varphi_{v 0}, \varphi_{v 1}$ and $\varphi_{v 3}$ 


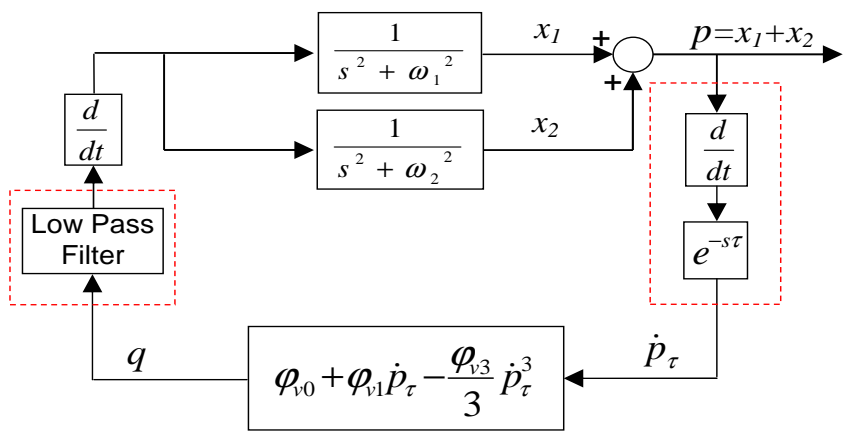

Fig. 2. Proposed combustion instability model

are arbitrary constants with the condition that $\varphi_{v 1}$ and $\varphi_{v 3}$ have the same sign. This allows accurate approximation of the static characteristic identified from experiments [1]. The coexistence of two oscillating modes can be explained when a time delay plus differentiator block and a low pass filter are included in the feedback path of the model (in dashed boxes). The modifications lead to the model presented in Figure 2 and is described by the following equations

$$
\left\{\begin{array}{l}
\ddot{x}_{1}+\omega_{1}^{2} x_{1}=\frac{d}{d t} L P F\left\{\varphi_{v 0}+\varphi_{v 1} \dot{p}_{\tau}-\frac{\varphi_{v 3}}{3} \dot{p}_{\tau}^{3}\right\}, \\
\ddot{x}_{2}+\omega_{2}^{2} x_{2}=\frac{d}{d t} L P F\left\{\varphi_{v 0}+\varphi_{v 1} \dot{p}_{\tau}-\frac{\varphi_{v 3}}{3} \dot{p}_{\tau}^{3}\right\},
\end{array}\right.
$$

where $\tau$ is a transport time delay from nozzle to flame surface, $L P F$ is the transfer operator of the low pass filter, $p=x_{1}+x_{2}$ is the downstream pressure perturbation at the burning plane, $\dot{p}_{\tau}$ is the output of the delay-plusdifferentiator block and $q$ is the flame heat release rate.

The new model is more complex and needs to be analyzed by an efficient approximation method as Krylov-Bogoliubov (K-B) approximation detailed in [18], [19], [20], [21], [22]. The presence of delay and low pass filtering yields some difficulties in computing approximations, which will be treated in this paper by introducing some realistic assumptions which are effective in stationary regime. Consequently, our approximations will be powerful at stationary regime. Also, to perform the approximations, we will use the refined K-B procedure which will add multiple harmonics.

There is a long history of modeling this instability, which is well summarized in [9]. Modeling splits into two main approaches; Galerkin approximation of partial-differentalequation descriptions based on physics, and black-box system identification methods which do not account for physics other than through their structure. The authors of [9] then move on to consider control approaches with a number of different schemes and the experimental performance. Some of these are model-based and others adapt a single control parameter directly. In no case is the control design nonlinear and attempt to benefit from the nature of the model. Here, we develop and analyze a model based on a variation of that from [1] and establish: that it is capable of demonstrating the salient dynamical features of the instability and its control, and that it is amenable to analysis using K-B methods. The contribution is thus in the tractability of the model class for control design.

\section{REFINED K-B APPROXIMATION: INTRODUCING NEGLECTED HARMONICS}

Consider a system with $n$ resonators described by differential equations of the form

$$
\ddot{x}_{k}+\omega_{k}^{2} x_{k}=\epsilon f_{k}(x, \dot{x}), \quad(k=1,2, \ldots, n)
$$

where $x=\left\{x_{1}, \ldots, x_{n}\right\}, \dot{x}=\left\{\dot{x_{1}}, \ldots, \dot{x}_{n}\right\}$ and $\epsilon$ is a small parameter.

In the previous article [17], we have given a summary of classical first K-B approximation, which consists of using only the fundamental terms in $f_{k}$. However, in some cases the outputs $x_{k}$ contain other harmonics with non negligible amplitudes (with respect to fundamental harmonic). These harmonics correspond to the other terms initially neglected in $f_{k}$ and will now be considered.

Rewrite the solution in another form

$$
x_{k}=a_{k} \cos \left(\psi_{k}\right)+u_{k}
$$

where; $\psi_{k}=\omega_{k} t+\theta_{k}, u_{k}$ is a sum of periodic functions excluding the fundamental harmonic, $a_{k}$ and $\theta_{k}$ are slowly time-varying functions, obeying the following equations

$$
\begin{aligned}
& \left\{\begin{array}{c}
\dot{a}_{k}=-\frac{\epsilon}{2 \omega_{k}} H_{k k}\left(a_{1}, \ldots, a_{n}, \theta_{1}, \ldots, \theta_{n}\right), \\
\dot{\theta}_{k}=-\frac{\epsilon}{2 \omega_{k} a_{k}} G_{k k}\left(a_{1}, \ldots, a_{n}, \theta_{1}, \ldots, \theta_{n}\right),
\end{array}\right. \\
& u_{k}=\sum_{\omega_{k} \neq \omega_{\ell}}^{r} \frac{H_{\ell k} \sin \left(\omega_{\ell} t+\theta_{\ell}\right)+G_{\ell k} \cos \left(\omega_{\ell} t+\theta_{\ell}\right)}{w_{k}^{2}-w_{\ell}^{2}},
\end{aligned}
$$

where $H_{k k}, G_{k k}, H_{\ell k}$ and $G_{\ell k}$ are obtained from the function $f_{k}$ by substituting

$$
\left\{\begin{array}{l}
x_{k}=a_{k} \cos \left(\omega_{k} t+\theta_{k}\right), \\
\dot{x}_{k}=-a_{k} \omega_{k} \sin \left(\omega_{k} t+\theta_{k}\right),
\end{array} \quad(k=1,2, \ldots, n)\right.
$$

and by setting it in the form

$$
\begin{aligned}
& f_{k}\left(a_{1} \cos \left(\omega_{1} t+\theta_{1}\right), \ldots, a_{n} \cos \left(\omega_{n} t+\theta_{n}\right),\right. \\
& \left.\quad \quad-a_{1} \omega_{1} \sin \left(\omega_{1} t+\theta_{1}\right), \ldots,-a_{n} \omega_{n} \sin \left(\omega_{n} t+\theta_{n}\right)\right) \\
& =H_{k k} \sin \left(\omega_{k} t+\theta_{k}\right)+G_{k k} \cos \left(\omega_{k} t+\theta_{k}\right) \\
& +\sum_{\omega_{k} \not \omega_{\ell}}^{r}\left(H_{\ell k} \sin \left(\omega_{\ell} t+\theta_{\ell}\right)+G_{\ell k} \cos \left(\omega_{\ell} t+\theta_{\ell}\right)\right),
\end{aligned}
$$

where $\omega_{\ell}$ and $\theta_{\ell}$ are the linear combinations of $\omega_{1}, \ldots, \omega_{n}$ and $\theta_{1}, \ldots, \theta_{n}$, respectively, and $r$ is the number of possible linear combinations of $\omega_{1}, \ldots, \omega_{n}$ different from $\omega_{k}$.

\section{EQUATION DEVELOPMENT AND ANALYSIS}

Consider the system (1) and the form (2) (with $\epsilon=1$ ), in this case

$$
f_{1}=f_{2}=\frac{d}{d t} \operatorname{LPF}\left\{\varphi_{v 0}+\varphi_{v 1} \dot{p}_{\tau}-\frac{\varphi_{v 3}}{3} \dot{p}_{\tau}^{3}\right\} .
$$

Replacing

$$
\dot{x}_{i}=-a_{i} \omega_{i} \sin \left(\omega_{i} t+\theta_{i}\right),(i=1,2)
$$

in $\dot{p}$, one obtains

$$
\begin{aligned}
\dot{p} & =\dot{x_{1}}+\dot{x_{2}} \\
\Rightarrow \dot{p} & =-\omega_{1} a_{1} \sin \left(\omega_{1} t+\theta_{1}\right)-\omega_{2} a_{2} \sin \left(\omega_{2} t+\theta_{2}\right) \\
& =\omega_{1} a_{1} \cos \left(\omega_{1} t+\theta_{1}+\frac{\pi}{2}\right)+\omega_{2} a_{2} \cos \left(\omega_{2} t+\theta_{2}+\frac{\pi}{2}\right),
\end{aligned}
$$


which after adding delay effect, takes the form

$$
\begin{aligned}
\dot{p}_{\tau}= & \omega_{1} a_{1 \tau} \cos \left(\omega_{1} t+\theta_{1 \tau}+\frac{\pi}{2}-\omega_{1} \tau\right) \\
& +\omega_{2} a_{2 \tau} \cos \left(\omega_{2} t+\theta_{2 \tau}+\frac{\pi}{2}-\omega_{2} \tau\right),
\end{aligned}
$$

where $a_{1 \tau}, a_{2 \tau}, \theta_{1 \tau}$ and $\theta_{2 \tau}$ are amplitudes and phases after the delay block, respectively. Since, for K-B approximation the amplitudes $a_{i}$ and the phases $\theta_{i}(i=1,2)$ are slowly time-varying functions, and in order to approximate the time delay block, we propose the following assumption.

Assumption 1: For small time delay $\tau$, the quantities $\left|a_{i}-a_{i \tau}\right|$ and $\left|\theta_{i}-\theta_{i \tau}\right|(i=1,2)$ can be neglected.

The assumption 1 allows the following approximations

$$
\left\{\begin{array}{l}
a_{i \tau}=a_{i}-\left(a_{i}-a_{i \tau}\right) \approx a_{i}, \quad(i=1,2) \\
\theta_{i \tau}=\theta_{i}-\left(\theta_{i}-\theta_{i \tau}\right) \approx \theta_{i} .
\end{array}\right.
$$

Substituting approximations (10) in expression (9), one gets

$$
\begin{aligned}
\dot{p}_{\tau} \approx \omega_{1} a_{1} \cos \left(\omega_{1} t+\theta_{1}+\frac{\pi}{2}-\omega_{1} \tau\right) & \\
& +\omega_{2} a_{2} \cos \left(\omega_{2} t+\theta_{2}+\frac{\pi}{2}-\omega_{2} \tau\right) .
\end{aligned}
$$

We use the following notation in the remaining of paper

$$
\psi_{k_{1} k_{2}}=\left(k_{1} \omega_{1}+k_{2} \omega_{2}\right) t+\left(k_{1} \theta_{1}+k_{2} \theta_{2}\right) .
$$

Introducing (11), using notation (12) in nonlinear static function and trigonometrical simplifications lead to the expression written in Appendix (A.25).

To get an expression in the form of (7), the low pass filter block must be also approximated. Therefore, we consider a second assumption

Assumption 2: The low pass filter is linear and the low pass filter dynamics are much faster than the evolution of amplitudes and phases.

The utility of assumption 2 is that, for an input given as the sum of sinusoidal terms (such as (A.25)), the output will be equal to the sum of the outputs of each term, and for sinusoidal inputs with slowly time-varying amplitudes and phases the rise time will be neglected, and the amplitudes and phases will be considered as constant parameters. Therefore, the assumption 2 leads the following approximation

$$
\operatorname{LPF}(a \cos (\omega t+\theta)) \approx G(\omega) a \cos (\omega t+\theta-\phi(\omega))
$$

where $a, \omega$ and $\theta$ are the amplitude, the frequency and the phase of input, respectively, $G(\omega)$ and $\phi(\omega)$ are the gain and the phase at frequency $\omega$ introduced by the filter. We use the following notation in the remaining of paper

$$
\begin{aligned}
& A_{k_{1} k_{2}}=\omega_{1}^{\left|k_{1}\right|} \omega_{2}^{\left|k_{2}\right|} G\left(k_{1} \omega_{1}+k_{2} \omega_{2}\right), \\
& \chi_{k_{1} k_{2}}=\frac{\left(k_{1}+k_{2}\right) \pi}{2}-\left(k_{1} \omega_{1}+k_{2} \omega_{2}\right) \tau-\phi\left(k_{1} \omega_{1}+k_{2} \omega_{2}\right) .
\end{aligned}
$$

Using (A.25), property (13) and notation (14), one arrives at (B.26). Result (B.26) yields the frequency set

$$
\begin{aligned}
W= & \left\{\omega_{1}, \omega_{2}, 3 \omega_{1}, 3 \omega_{2}, 2 \omega_{1}+\omega_{2}, \omega_{1}+2 \omega_{2},\right. \\
& \left.2 \omega_{1}-\omega_{2}, 2 \omega_{2}-\omega_{1}\right\},
\end{aligned}
$$

which will be very important for identifying the different situations depending on the proximity of frequencies in $W$. Consequently, one has the following three situations:

1) $\omega_{1} \not \approx\left\{\omega_{2}, 3 \omega_{2}, \frac{\omega_{2}}{3}\right\}$,
2) $\omega_{1} \approx \omega_{2}:$ Mutual synchronization with close frequencies

3) $\omega_{1} \approx 3 \omega_{2}$ (respectively $\left.\omega_{2} \approx 3 \omega_{1}\right)$ : Mutual synchronization with multiple frequencies

Experimental results ([1]) reveal that both modes oscillate freely without synchronization and with the frequencies not respecting the conditions of cases number 2 and 3. Hence, we limit our study here to case 1 . This is new and more practically interesting when compared with [17].

\section{K-B APPROXIMATION OF THE MODEL}

Consider the condition $\omega_{1} \not \approx\left\{\omega_{2}, 3 \omega_{2}, \frac{\omega_{2}}{3}\right\}$ and the result (B.26). The application of refined K-B approximation gives

$$
x_{i}=a_{i} \cos \left(\omega_{i} t+\theta_{i}\right)+u_{i}, \quad(i=1,2)
$$

with

$\left\{\begin{array}{l}\dot{a}_{1}=\frac{\varphi_{v 1} A_{10} \cos \left(\chi_{10}\right)}{2} a_{1}\left(1-\frac{\varphi_{v 3}}{\varphi_{v 1}}\left(\frac{\left(\omega_{1} a_{1}\right)^{2}}{4}+\frac{\left(\omega_{2} a_{2}\right)^{2}}{2}\right)\right), \\ \dot{a}_{2}=\frac{\varphi_{v 1} A_{01} \cos \left(\chi_{01}\right)}{2} a_{2}\left(1-\frac{\varphi_{v 3}}{\varphi_{v 1}}\left(\frac{\left(\omega_{2} a_{2}\right)^{2}}{4}+\frac{\left(\omega_{1} a_{1}\right)^{2}}{2}\right)\right), \\ \dot{\theta}_{1}=\frac{\varphi_{v 1} A_{10} \sin \left(\chi_{10}\right)}{2}\left(1-\frac{\varphi_{v 3}}{\varphi_{v 1}}\left(\frac{\left(\omega_{1} a_{1}\right)^{2}}{4}+\frac{\left(\omega_{2} a_{2}\right)^{2}}{2}\right)\right), \\ \dot{\theta}_{2}=\frac{\varphi_{v 1} A_{01} \sin \left(\chi_{01}\right)}{2}\left(1-\frac{\varphi_{v 3}}{\varphi_{v 1}}\left(\frac{\left(\omega_{2} a_{2}\right)^{2}}{4}+\frac{\left(\omega_{1} a_{1}\right)^{2}}{2}\right)\right),\end{array}\right.$

where $u_{i}$ is given in appendix C. From the equations (17), one can see that the coupled parameters are $a_{1}$ and $a_{2}$. Therefore, the system dynamics depend essentially on the evolution of amplitudes $a_{1}$ and $a_{2}$. The analytical determination of equilibrium points gives

$$
\begin{gathered}
a_{1}=0 \text { and } a_{2}=0, \\
a_{1}=\frac{2}{\omega_{1}} \sqrt{\frac{\varphi_{v 1}}{\varphi_{v 3}}} \text { and } a_{2}=0, \\
a_{1}=0 \text { and } a_{2}=\frac{2}{\omega_{2}} \sqrt{\frac{\varphi_{v 1}}{\varphi_{v 3}}}, \\
a_{1}=\frac{2}{\omega_{1}} \sqrt{\frac{\varphi_{v 1}}{3 \varphi_{v 3}}} \text { and } a_{2}=\frac{2}{\omega_{2}} \sqrt{\frac{\varphi_{v 1}}{3 \varphi_{v 3}}} .
\end{gathered}
$$

The stability of each equilibrium point leads to a particular regime. Consequently, one distinguishes four operation regimes, which will be elaborated and explained shortly :

1) Asymptotically stable system,

2) Two generators with competitive quenching,

3) Simultaneous self-sustained oscillations,

4) Total instability.

For stability study one can apply Lyapunov's indirect method, which uses the stability property of linearized system around the equilibrium point. The computation of general characteristic polynomial leads to the result showed in Appendix D. The characteristic polynomial obtained is second order. Therefore, the stability can be verified by testing the signs of polynomial coefficients.

\section{A. Asymptotically stable system}

The system is asymptotically stable around the origin, if and only if the equilibrium point (18) is asymptotically stable. Introducing (18) in the general characteristic polynomial (D.28), one obtains

$$
\begin{aligned}
P(\lambda)= & \lambda^{2}-\frac{\varphi_{v 1}}{2}\left\{A_{10} \cos \left(\chi_{10}\right)+A_{01} \cos \left(\chi_{01}\right)\right\} \lambda \\
& +\frac{A_{10} A_{01} \cos \left(\chi_{10}\right) \cos \left(\chi_{01}\right) \varphi_{v 1}^{2}}{4},
\end{aligned}
$$



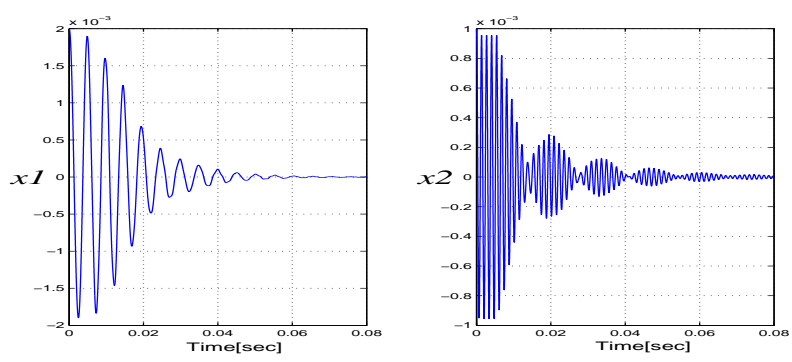

Fig. 3. Simulation test for $\omega_{1}=2 \pi \times 210, \omega_{2}=2 \pi \times 740, \varphi_{v 0}=$ $0.45, \varphi_{v 1}=-0.135, \varphi_{v 3}=-5.4 \times 10^{-3}, L P F=\frac{2 \pi \times 500}{s+2 \pi \times 500}$ and $\tau=5.5 \times 10^{-3}$.
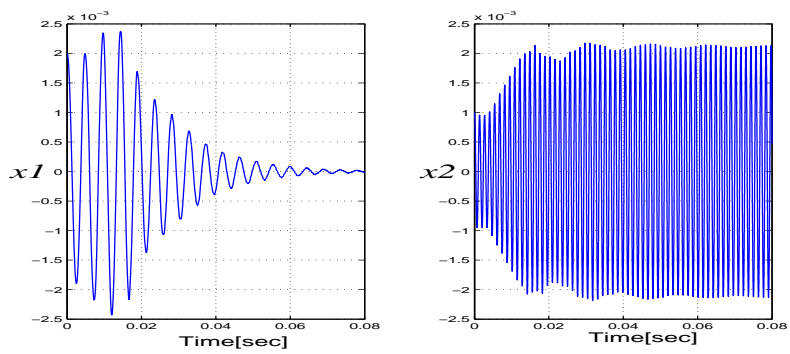

Fig. 4. Simulation test for $\omega_{1}=2 \pi \times 210, \omega_{2}=2 \pi \times 740, \varphi_{v 0}=$ $0.45, \varphi_{v 1}=-0.135, \varphi_{v 3}=-5.4 \times 10^{-3}, L P F=\frac{2 \pi \times 500}{s+2 \pi \times 500}$ and $\tau=3.5 \times 10^{-3}$.

which has two stable zeros if the following conditions are respected

$$
\begin{aligned}
\left\{\begin{array}{l}
-\varphi_{v 1}\left\{A_{10} \cos \left(\chi_{10}\right)+A_{01} \cos \left(\chi_{01}\right)\right\}>0 \\
A_{10} A_{01} \cos \left(\chi_{10}\right) \cos \left(\chi_{01}\right) \varphi_{v 1}^{2}>0
\end{array}\right. \\
\Longleftrightarrow\left\{\begin{array}{l}
\varphi_{v 1} \cos \left(\chi_{10}\right) \\
\varphi_{v 1} \cos \left(\chi_{01}\right)
\end{array}<0\right.
\end{aligned}
$$

Provided (22) is satisfied and initial states are close to the origin, the amplitudes of both oscillations converge to equilibrium point (18). Figure 3 shows an example of simulation test, where the conditions (22) are satisfied under realistic parameters values.

\section{B. Two generators with competitive quenching}

The two generators with competitive quenching regime occurs when both equilibrium points (19) and (20) are locally stable. Substituting (19) into (D.28), one gets

$$
\begin{aligned}
P(\lambda)= & \lambda^{2}+\varphi_{v 1}\left\{A_{10} \cos \left(\chi_{10}\right)+\frac{1}{2} A_{01} \cos \left(\chi_{01}\right)\right\} \lambda \\
& +\frac{A_{10} A_{01} \cos \left(\chi_{10}\right) \cos \left(\chi_{01}\right) \varphi_{v 1}^{2}}{2},
\end{aligned}
$$

The local stability of (19) is satisfied if and only if

$$
\begin{aligned}
\left\{\begin{array}{l}
\varphi_{v 1}\left\{A_{10} \cos \left(\chi_{10}\right)+\frac{1}{2} A_{01} \cos \left(\chi_{01}\right)\right\}>0 \\
A_{10} A_{01} \cos \left(\chi_{10}\right) \cos \left(\chi_{01}\right) \varphi_{v 1}^{2}>0
\end{array}\right. \\
\Longleftrightarrow\left\{\begin{array}{l}
\varphi_{v 1} \cos \left(\chi_{10}\right) \\
\varphi_{v 1} \cos \left(\chi_{01}\right)
\end{array}\right\rangle 0
\end{aligned}
$$

By symmetry, for the equilibrium point (20) one finds the same conditions.
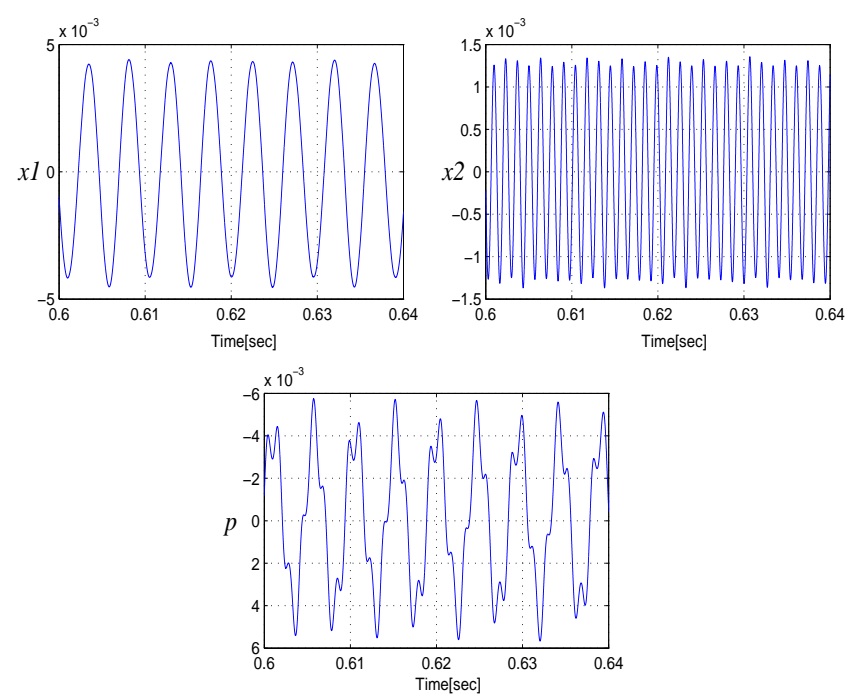

Fig. 5. Simulation test for $\omega_{1}=2 \pi \times 210, \omega_{2}=2 \pi \times 740, \varphi_{v 0}=$ $0.45, \varphi_{v 1}=-0.135, \varphi_{v 3}=-5.4 \times 10^{-3}, L P F=\frac{2 \pi \times 500}{s+2 \pi \times 500}$ and $\tau=4.8 \times 10^{-3}$.

Provided that the conditions (23) are satisfied, the amplitudes of $x_{1}$ and $x_{2}$ converge to one of both possible equilibrium points (19) and (20). Depending on the initial states, one of the generators is excited, while the oscillations of the other generator are entirely quenched. Figure 4 shows an example of simulation test on two generators with competitive quenching regime.

\section{Simultaneous self-sustained oscillations}

Simultaneous oscillation of both resonators occurs when the equilibrium point (21) is stable. Introducing (21) in (D.28), one obtains

$$
\begin{aligned}
P(\lambda)= & \lambda^{2}+\frac{\varphi_{v 1}}{3}\left\{A_{10} \cos \left(\chi_{10}\right)+A_{01} \cos \left(\chi_{01}\right)\right\} \lambda \\
& -\frac{A_{10} A_{01} \cos \left(\chi_{10}\right) \cos \left(\chi_{01}\right) \varphi_{v 1}^{2}}{3},
\end{aligned}
$$

The equilibrium point (21) is locally stable if and only if

$$
\begin{gathered}
\left\{\begin{array}{l}
\varphi_{v 1}\left\{A_{10} \cos \left(\chi_{10}\right)+A_{01} \cos \left(\chi_{01}\right)\right\}>0 \\
\left.-A_{10} A_{01} \cos \left(\chi_{10}\right) \cos \left(\chi_{01}\right) \varphi_{v 1}^{2}\right\rangle 0
\end{array}\right. \\
\Longleftrightarrow\left\{\begin{array}{l}
\varphi_{v 1}\left(A_{10} \cos \left(\chi_{10}\right)+A_{01} \cos \left(\chi_{01}\right)\right)>0 \\
\cos \left(\chi_{10}\right) \cos \left(\chi_{01}\right)\langle 0
\end{array}\right.
\end{gathered}
$$

By satisfying (24), it is possible to have simultaneous selfsustained oscillations, the amplitudes of $x_{1}$ and $x_{2}$ converge to the equilibrium point (21). By the self-sustained oscillations it is meant that both oscillators are excited without synchronization. The Figure 5 shows the stationary part of a simulation test example on simultaneous self-sustained oscillations regime.

\section{Total instability}

When the conditions (22), (23) and (24) are not satisfied, there does not exist a stable equilibrium point. Therefore, there is no stable limit cycle and the amplitudes of both oscillations diverge. By total instability it is meant that for any initial states, the oscillations of the system diverge. 


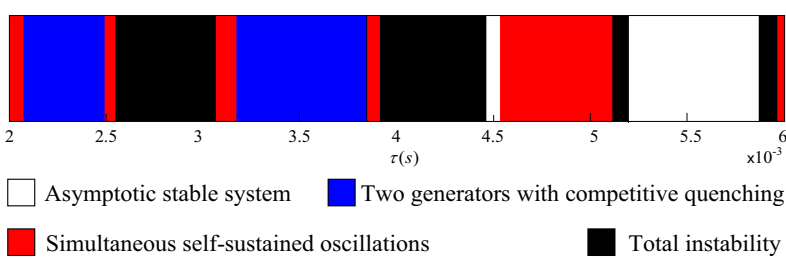

Fig. 6. The operation regimes as a function of $\tau, \omega_{1}=2 \pi \times 210$, $\omega_{2}=2 \pi \times 740, \varphi_{v 0}=0.45, \varphi_{v 1}=-0.135, \varphi_{v 3}=-5.4 \times 10^{-3}$ and $L P F=\frac{2 \pi \times 500}{s+2 \pi \times 500}$.

\section{RESUlTS ANALYSis}

The results demonstrate the existence of four operation regimes when the ratio of natural frequencies is different from 1,3 and $\frac{1}{3}$. The occurrence of each operation regime depends on the satisfaction of some conditions. The simulations confirm the quality of estimated amplitudes using K-B approximation. The condition for each regime contain essentially the phases $\chi_{01}$ and $\chi_{10}$ introduced by the delay and low pass filtering respectively. The phase domain conditions (22), (23) and (24) are independent. So with fixed delay and low-pass filter it is not possible to have operation in more than one regime. The results suggest that it is perhaps possible in certain cases to estimate the delay from the measurement of the oscillations (number, frequency, amplitude). To illustrate the importance of delay, Figure 6 depicts the operation regimes for several values of $\tau$ and for other parameters fixed near to the practical values. One observes the occurrence of various regimes as a function of the delay.

From a practical point of view, the interesting situation is the simultaneous self-sustained oscillatory regime which is represented in Figure 6 in red. The fact that, the amplitudes of harmonics $\omega_{1}$ and $\omega_{2}$ take values $\frac{2}{\omega_{1}} \sqrt{\frac{\varphi_{v 1}}{3 \varphi_{v 3}}}$ and $\frac{2}{\omega_{2}} \sqrt{\frac{\varphi_{v 1}}{3 \varphi_{v 3}}}$ respectively, shows clearly that they depend inversely on the values of frequencies (phenomenon which has been observed in practice). The refined K-B procedure added the multiple harmonics of $\omega_{1}$ and $\omega_{2}$ to the approximations. The evaluation of the precision of the refined K-B approximations requires a spectral comparison with the real outputs of the model. The stationary power spectral density comparison using FFT is presented in Figure 7, where $D_{x 1}, D_{x 2}$ and $D_{p}$ are the power spectral densities of $x_{1}, x_{2}$ and $p$, respectively. From Figure 7, one can see that the important harmonics of the model outputs have been captured with an excellent approximation of the gains. The other uncaptured harmonics have negligible gains.

\section{COMPARISON WITH EXPERIMENTAL RESULTS}

The capacity of a combustion instability model to display the coexistence of two modes is regarded as an important corroboration of the model. Indeed, the representative experimental results discussed in [2], [16] demonstrate the simultaneous presence of two sinusoidal components; a strong dominant tone at $210 \mathrm{~Hz}$, and a weak but persistent nonharmonic tone at $714 \mathrm{~Hz}$.
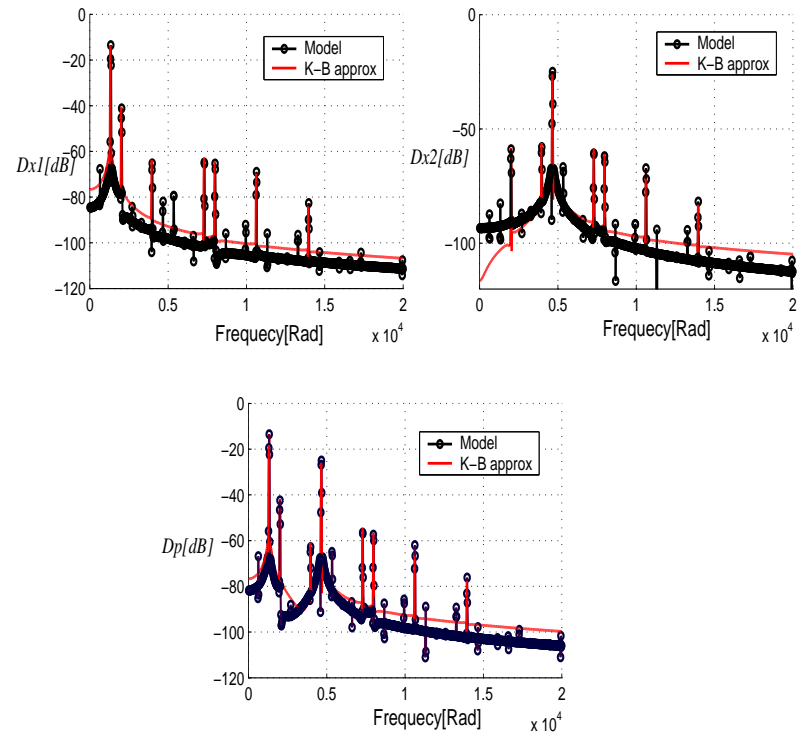

Fig. 7. Spectral comparison of real and approximated outputs for $\omega_{1}=$ $2 \pi \times 210, \omega_{2}=2 \pi \times 740, \varphi_{v 0}=0.45, \varphi_{v 1}=-0.135, \varphi_{v 3}=-5.4 \times$ $10^{-3}, L P F=\frac{2 \pi \times 500}{s+2 \pi \times 500}$ and $\tau=4.8 \times 10^{-3}$.

The model proposed here has the ability to describe a number of distinct phenomena observed in practice. One of most important is simultaneous self-sustained oscillations phenomenon which occurs when the conditions (24) are satisfied. The analysis of this phenomenon shows that, on one hand, the model can display the coexistence of two modes at any frequencies (depending of the value of the delay), and on other hand it shows that the amplitudes of both oscillations decrease when the frequency increase. As the analysis has shown, the model can display also the other situations encountered in practice.

The coherence of the theoretical analysis with the experimental and model simulation results constitutes also an important fact, indicating that K-B method is a powerful analysis method for the combustion instability.

\section{CONCLUSION}

This article focused on proposing and analyzing an attractive dynamic model for combustion instability having a feedback structure. The crucial role of the delay in the feedback path of the model has been emphasized. The important consequence is that the new model captures the coexistence of two modes phenomena, which occur in real systems. The efficiency of refined K-B analysis method has been illustrated by a spectral comparison of the true model outputs with those of the computed approximations.

One of the major results is the demonstration of occurrence of simultaneous self-sustained oscillations which have a frequency ratio different from 1,3 and $\frac{1}{3}$ and with a magnitude decreasing with the frequency. Furthermore, a thorough full analysis of the effect of the delay has been provided showing clearly the possibility of occurrence of various operation regimes as a function of the value of the delay. This may 
constitute also a starting point for an estimation of delay from experimental data.

Further work will focus on the establishment of conditions for the quenching of the oscillations by adding an external modulation of a fraction of the fuel flow into the combustion chamber.

\section{REFERENCES}

[1] W. J. Dunstan, "System identification of nonlinear resonant systems," Ph.D. dissertation, University of California, San Diego, 2003.

[2] R. M. Murray, C. A. Jacobson, R. Casas, A. I. Khibnik, C. R. Johnson Jr, R. R. Bitmead, A. A. Peracchio, and W. M. Proscia, "System identification for limit cycling systems: a case study for combustion instabilities," American Control Conference, Philadelphia PA, pp. 2004-2008, 1998.

[3] V. Faivre and T. Poinsot, "Experimental and numerical investigations of jet active control for combustion applications," Journal of Turbulence, vol. 5, no. 025, Aug 2004.

[4] S. R. Stow and A. Dowling, "Low-ordre modelling of thermoacoustic limit cycles," Proceedings of ASME TURBO EXPO, Jun 2004.

[5] C. Jacobson, A. Khibnik, A. Banaszuk, J. Cohen, and W. Proscia, "Active control of combustion instabilities in gas turbine engines for low emissions. part I: Physics-based and experimentally identified models of combustion instability," Proc. AVT Symposium on Active Control Technology, Braunschweig, pp. 30-1-30-11, 2000.

[6] K. McManus, F. Han, W. Dunstan, C. Barbu, and M. Shah, "Modeling and control of combustion dynamics in industrial gas turbines," Proc. ASME Turbo-Expo, pp. 567-575, 2004.

[7] S. Candel, "Combustion dynamics and control: progress and challenges," Proceedings of the Combustion Institute, vol. 29, pp. 1-28, 2002.

[8] N. Docquier and S. Candel, "Combustion control and sensors: a review," Progress in Energy and Combustion Science, vol. 28, pp. 107-150, 2002.

[9] A. Annaswamy and A. Ghoniem, "Active control of combustion instability: Theory and practice," IEEE Control Systems Magazine, vol. 22, no. 6, pp. 37-54, Dec 2002.

[10] G. Roy, Advances in Chemical Propulsion: Science to Technology. Boc Raton, Fl USA: CRC - Taylor \& Francis, 2001.

[11] A. Banaszuk, K. Ariyur, M. Krstic, and C. Jacobson, "An adaptive algorithm for control of combustion instability," Automatica, vol. 40, pp. 1965-1972, 2004.

[12] J. Rayleigh, The Theory of Sound. New York: Dover, 1945, vol. 2.

[13] P. Rijke, Ann. of Physics, 1959.

[14] J. Tyndal, Sound. New York: D. Appleton \& Company, 1897.

[15] A. Peracchio and W. Proscia, "Nonlinear heat-release/acoustic model for thermoacoustic instability in lean premixed combustors," ASME J. Eng. Gas Turbines Power, vol. 121, no. 3, pp. 415-421, 1999.

[16] W. J. Dunstan, R. R. Bitmead, and S. M. Savaresi, "Fitting nonlinear low-order models for combustion instability control," Control Engineering Practice, pp. 1301-1317, 2001.

[17] F. Bouziani, I. D. Landau, R. R. Bitmead, and A. Voda-Besançon, "An analytically tractable model for combustion instability," $C D C-E C C$, no. 44, 2005.

[18] N. Bogoliubov and Y. Mitropolski, Asymptotic Methods in the Theory of Nonlinear Oscillations. New York: Hindustan Publishing Corp, Delhi, and Gordon and Breach, 1961.

[19] C. Hayashi, Nonlinear Oscillations in Physical Systems. New York: McGraw-Hill Book Co, 1964, (reprinted by Princeton University Press, 1985).

[20] P. S. Landa, Nonlinear Oscillations and Waves in Dynamical Systems. Kluwer, 1996.

[21] _ Regular and Chaotic Oscillations, V. I. Babitsky and J. Wittenburg, Eds. New York: Springer, 2000.

[22] I. D. Landau and R. R. Bitmead, "On the method of Krylov and Bogoliubov for the analysis of nonlinear oscillations," Mechanical and Aerospace Engineering Department, University of California, San Diego, 9500 Gilman Drive, La Jolla CA 92093-0411, USA, Tech. Rep., Jan 2004.
APPENDIX A

\section{ASSUMPTION 1 CONSEQUENCE}

$\varphi_{v 0}+\varphi_{v 1} \dot{p}_{-\tau}-\frac{\varphi_{v 3}}{3} \dot{p}_{-\tau}^{3} \approx \varphi_{v 0}+\varphi_{v 1}\left\{\omega_{1} a_{1}\left(1-\frac{\varphi_{v 3}}{\varphi_{v 1}}\right.\right.$ $\left.\left(\frac{\left(\omega_{1} a_{1}\right)^{2}}{4}+\frac{\left(\omega_{2} a_{2}\right)^{2}}{2}\right)\right) \cos \left(\psi_{10}+\frac{\pi}{2}-\omega_{1} \tau\right)+\omega_{2} a_{2}(1$ $\left.\left.-\frac{\varphi_{v 3}}{\varphi_{v 1}}\left(\frac{\left(\omega_{2} a_{2}\right)^{2}}{4}+\frac{\left(\omega_{1} a_{1}\right)^{2}}{2}\right)\right) \cos \left(\psi_{01}+\frac{\pi}{2}-\omega_{2} \tau\right)\right\}-\varphi_{v 3}$ $\left\{\frac{\left(\omega_{1} a_{1}\right)^{3}}{12} \cos \left(\psi_{30}+\frac{3 \pi}{2}-3 \omega_{1} \tau\right)+\frac{\left(\omega_{2} a_{2}\right)^{3}}{12} \cos \left(\psi_{03}+\frac{3 \pi}{2}\right.\right.$ $\left.-3 \omega_{2} \tau\right)+\frac{\omega_{1}^{2} \omega_{2} a_{1}^{2} a_{2}}{4} \cos \left(\psi_{21}+\frac{3 \pi}{2}-\left(2 \omega_{1}+\omega_{2}\right) \tau\right)$ $+\frac{\omega_{1} \omega_{2}^{2} a_{1} a_{2}^{2}}{4} \cos \left(\psi_{12}+\frac{3 \pi}{2}-\left(2 \omega_{2}+\omega_{1}\right) \tau\right)$ $+\frac{\omega_{1}^{2} \omega_{2} a_{1}^{2} a_{2}}{4} \cos \left(\psi_{2-1}+\frac{\pi}{2}-\left(2 \omega_{1}-\omega_{2}\right) \tau\right)$ $\left.+\frac{\omega_{1} \omega_{2}^{2} a_{2}^{2} a_{1}}{4} \cos \left(\psi_{-12}+\frac{\pi}{2}-\left(2 \omega_{2}-\omega_{1}\right) \tau\right)\right\}$.

\section{APPENDIX B}

\section{ASSUMPTION 2 CONSEQUENCE}

$$
\begin{aligned}
& \frac{d}{d t} \operatorname{LPF}\left\{\varphi_{v 0}+\varphi_{v 1} \dot{p}_{-\tau}-\frac{\varphi_{v 3}}{3} \dot{p}_{-\tau}^{3}\right\} \approx-\varphi_{v 1}\left\{\omega_{1} A_{10} a_{1}(1\right. \\
& \left.-\frac{\varphi_{v 3}}{\varphi_{v 1}}\left(\frac{\left(\omega_{1} a_{1}\right)^{2}}{4}+\frac{\left(\omega_{2} a_{2}\right)^{2}}{2}\right)\right) \sin \left(\psi_{10}+\chi_{10}\right)+\omega_{2} A_{01} a_{2}(1 \\
& \left.\left.-\frac{\varphi_{v 3}}{\varphi_{v 1}}\left(\frac{\left(\omega_{2} a_{2}\right)^{2}}{4}+\frac{\left(\omega_{1} a_{1}\right)^{2}}{2}\right)\right) \sin \left(\psi_{01}+\chi_{01}\right)\right\}+\varphi_{v 3}\{ \\
& \frac{\omega_{1} A_{30} a_{1}^{3}}{4} \sin \left(\psi_{30}+\chi_{30}\right)+\frac{\omega_{2} A_{03} a_{2}^{3}}{4} \sin \left(\psi_{03}+\chi_{03}\right) \\
& +\frac{\left(2 \omega_{1}+\omega_{2}\right) A_{21} a_{1}^{2} a_{2}}{4} \sin \left(\psi_{21}+\chi_{21}\right)+\frac{\left(\omega_{1}+2 \omega_{2}\right) A_{12} a_{1} a_{2}^{2}}{4} \\
& \sin \left(\psi_{12}+\chi_{12}\right)+\frac{\left(2 \omega_{1}-\omega_{2}\right) A_{2-1} a_{1}^{2} a_{2}}{4} \sin \left(\psi_{2-1}+\chi_{2-1}\right) \\
& \left.+\frac{\left(2 \omega_{2}-\omega_{1}\right) A_{-12} a_{2}^{2} a_{1}}{4} \sin \left(\psi_{-12}+\chi_{-12}\right)\right\} .
\end{aligned}
$$

\section{APPENDIX C}

\section{REFINEMENT COMPONENT}

$$
\begin{aligned}
& u_{i}=-\frac{\varphi_{v 1} a_{3-i} \omega_{3-i} A_{i-1} 2-i}{\omega_{i}^{2}-\omega_{3-i}^{2}}\left(1-\frac{\varphi_{v 3}}{\varphi_{v 1}}\left(\frac{\left(\omega_{3-i} a_{3-i}\right)^{2}}{4}+\frac{\left(\omega_{i} a_{i}\right)^{2}}{2}\right.\right. \\
& )) \sin \left(\psi_{i-1} 2-i+\chi_{i-1} 2-i\right)+\varphi_{v 3}\left\{\frac { \omega _ { 1 } A _ { 3 0 } a _ { 1 } ^ { 3 } } { 4 ( \omega _ { i } ^ { 2 } - 9 \omega _ { 1 } ^ { 2 } ) } \operatorname { s i n } \left(\psi_{30}\right.\right. \\
& \left.+\chi_{30}\right)+\frac{\omega_{2} A_{03} a_{2}^{3}}{4\left(\omega_{i}^{2}-9 \omega_{2}^{2}\right)} \sin \left(\psi_{03}+\chi_{03}\right)+\frac{\left(2 \omega_{1}+\omega_{2}\right) A_{21} a_{1}^{2} a_{2}}{4\left(\omega_{i}^{2}-\left(2 \omega_{1}+\omega_{2}\right)^{2}\right)} \\
& \sin \left(\psi_{21}+\chi_{21}\right)+\frac{\left(\omega_{1}+2 \omega_{2}\right) A_{12} a_{1} a_{2}^{2}}{4\left(\omega_{i}^{2}-\left(2 \omega_{2}+\omega_{1}\right)^{2}\right)} \sin \left(\psi_{12}+\chi_{12}\right) \\
& +\frac{\left(2 \omega_{1}-\omega_{2}\right) A_{2-1} a_{1}^{2} a_{2}}{4\left(\omega_{i}^{2}-\left(2 \omega_{1}-\omega_{2}\right)^{2}\right)} \sin \left(\psi_{2-1}+\chi_{2-1}\right) \\
& \left.\left.+\frac{\left(2 \omega_{2}-\omega_{1}\right) A_{-12} a_{2}^{2} a_{1}}{4\left(\omega_{i}^{2}-\left(2 \omega_{2}-\omega_{1}\right)^{2}\right)} \sin \left(\psi_{-12}+\chi_{-12}\right)\right\},(i=1,2) \quad \text { (C. } 27\right)
\end{aligned}
$$

\section{APPENDIX D}

\section{GENERAL CHARACTERISTIC POLYNOMIAL}

$$
\begin{aligned}
& P(\lambda)=\lambda^{2}-\frac{\varphi_{v 1}}{2}\left\{A _ { 1 0 } \operatorname { c o s } ( \chi _ { 1 0 } ) \left(1-\frac{\varphi_{v 3}}{\varphi_{v 1}}\left(\frac{3\left(\omega_{1} a_{1}\right)^{2}}{4}\right.\right.\right. \\
& \left.\left.+\frac{\left(\omega_{2} a_{2}\right)^{2}}{2}\right)\right)+A_{01} \cos \left(\chi_{01}\right)\left(1-\frac{\varphi_{v 3}}{\varphi_{v 1}}\left(\frac{3\left(\omega_{2} a_{2}\right)^{2}}{4}\right.\right. \\
& \left.\left.\left.+\frac{\left(\omega_{1} a_{1}\right)^{2}}{2}\right)\right)\right\} \lambda+\frac{A_{10} A_{01} \cos \left(\chi_{10}\right) \cos \left(\chi_{01}\right)}{4}\left\{\varphi_{v 1}^{2}(1\right. \\
& \left.-\frac{\varphi_{v 3}}{\varphi_{v 1}}\left(\frac{3\left(\omega_{1} a_{1}\right)^{2}}{4}+\frac{\left(\omega_{2} a_{2}\right)^{2}}{2}\right)\right)\left(1-\frac{\varphi_{v 3}}{\varphi_{v 1}}\left(\frac{3\left(\omega_{2} a_{2}\right)^{2}}{4}\right.\right. \\
& \left.\left.\left.+\frac{\left(\omega_{1} a_{1}\right)^{2}}{2}\right)\right)-\varphi_{v 3}^{2}\left(\omega_{1} \omega_{2} a_{1} a_{2}\right)^{2}\right\} .
\end{aligned}
$$

